\title{
A Tribute to Bohdan Paczyński (1940-2007)
}

\section{Shude Mao*}

Jodrell Bank Centre for Astrophysics, University of Manchester, Manchester M13 9PL, UK

E-mail: shude.mao@manchester.ac.uk

This conference was partly organised in memory of Professor Bohdan Paczyński who initiated the field of microlensing, and has made enormous contributions to astrophysics. Bohdan Paczyński is a unique scientist and a kind man who has impacted the research of many at the conference today. I share some personal memories of him as an ex-student and collaborator.

The Manchester Microlensing Conference: The 12th International Conference and ANGLES Microlensing Workshop

January 21-25 2008

Manchester, UK

${ }^{*}$ Speaker. 


\section{Introduction}

Such was Bohdan Paczyński 's impact on my career and my life that hardly a day passes without me thinking about him since his death on April 19, 2007. This conference is the 12th conference on gravitational microlensing, a field he initiated in 1986 [9]. There are a number of his students, intellectual grandchildren, postdocs, and collaborators in attendance at the conference today. So here we gather to celebrate his life and his contributions to astrophysics. The organisers asked me to pay a personal tribute him. I was his PhD student from 1990-1992, and visited him almost annually until 2003. It is not exaggerating to say that he showed me how to do science and how to be a human being. I owe a tremendous debt to him (and to many other people at Peyton Hall and Princeton University).

\section{Bohdan Paczyński as a unique scientist and a great man}

Bohdan was a great scientist. A search on the Astrophysics Data System ${ }^{1}$ found 353 publications (227 refereed), and more than 15000 citations. Even these impressive numbers do not fully convey the contributions he made to astrophysics, his originality and creativity.

He has made seminal contributions to many fields in astrophysics, including stellar evolution [6, 7], accretion disks [8], gravitational lensing (including microlensing [9, 3], and giant arcs by clusters of galaxies [11]), cosmology (the Alcock-Paczński test [1]), and of course gamma-ray bursts [10, 12]. His publication record spans for more than four decades (from 1965-2006), and, remarkably, he made major contributions to different fields in each decade. Because of his scientific contributions, he received many honors, including the Henry Russell Lectureship from the American Astronomical Society and the Gold medal from the Royal Astronomical Society.

Bohdan had probably the best intuition among the astronomers that I have met. He emphasized simple geometric pictures rather than complex mathematics, and had a remarkable ability to see through the "fogs" of observational data and grasp the essence of a problem, through "common sense" [2]. Being a starting PhD student was, however, occasionally a frustrating experience: just imagine yourself doing several days of calculations without making sense of the results, you went to Bohdan and then he instantly understood the results and explained to you why. Part of his intuition may come from experience, but I believe he has something else that one can only emulate but never quite learn. His lecture course on stellar astrophysics at Peyton hall is also an example of clarity and directness.

Bohdan's courage to go against the "bandwagon" is legendary. When I was leaving Princeton, I mentioned to him that I wanted to work on something other than gravitational lensing, for example cosmology. His instant reaction was, perhaps half-seriously, that "there are so many cosmologists - they must be missing something!". Another well-known example is his idea on the cosmological origin of gamma-ray bursts. For this talk, I checked the referee's reports for some of our joint papers. I started to appreciate much more the kind of convictions he needed to pursue his theory. For example, we wrote a paper [4] on the cosmological origin of gamma-ray bursts right after BATSE's discovery of their isotropic distributions in the sky [5]. The referee wrote "I have read the revised copy of the paper sent to me by Bohdan Paczynski. The errors which I pointed out in the original

\footnotetext{
${ }^{1}$ http://adswww.harvard.edu/
} 
version have been corrected. However, the authors still ignore the extensive spectral and temporal evidence which I mentioned, making veiled references to them only as "theoretical arguments" and "elaborate theoretical models." This seems to be as much as they can bring themselves to say. I don't think too many will be fooled by it, and it should amuse many others, so I am willing to recommend this version for publication." This illustrates not only the courage Bohdan had when he was going against the flow, but also the fact that at any given time, observational data are often ambiguous, and Bohdan was able to distinguish theoretical models ("fantasies") from reality.

He also had infectious enthusiasm. I still remember the few weeks we spent together on our (my) first paper. At that time, I was working on the statistics of galaxy-scale lenses. One day I was sitting in a chair just under the black board in his room (close to the door), he was sitting opposite to me, talking about various topics. At that time (at the beginning of 1991), cosmological microlensing was already established for the gravitational lens 2237+0305 (Huchra's lens), and we were discussing how to calculate the light curves more efficiently for microlensing at large optical depth as part of my $\mathrm{PhD}$ thesis (this is yet to be done!). At that time, microlensing surveys just started but has not yet discovered any events. He mentioned in passing that "who knows - microlensing may even be useful for finding extrasolar planets." Without saying anything, I went away and calculated the caustics and critical curves for a Jupiter-mass planet around a solartype star at one Einstein radius. When he saw the large, non-negligible size of the caustics, he immediately realised that this offers a new method of planet detection. He was so excited that he asked me to stop everything else, and we worked intensively on the paper [3], and finished the work in two or three weeks. unfortunately such a short timescale for writing a paper from the start to finish has never been repeated! I always remembered the referee's report being very positive, but when I checked the record, this was not entirely the case. The referee wrote "This paper deserves publication in the Astrophysical Journal Letters. Much effort is currently being expended on projects to seek microlensing events of LMC and bulge stars by light and dark stellar mass lenses in a galaxy and it is incumbent upon theorists to provide the best guidance (even if this theorist is extremely skeptical that any convincing examples of gravitational lensing will be found in this way). The paper is clear and to the point and does not understate the difficulties. Curiously, it makes no mention of the possibility that the source may just as well be binary and that this, too, can produce more complicated variation than single star sources and lenses." At the time, the referee was not in the minority in the scepticism about the detection of microlensing events because of possible confusions with variable stars. In fact, this scepticism was widely shared at the Harvard-Smithsonian Center for Astrophysics when I was a postdoc there (1992-1995).

Bohdan was modest and extremely generous, freely giving away his ideas, and in his early days, a computer code on stellar evolution, to colleagues and students. I am sure many in the audience today had first-hand experience with his generosity. He took quite some pride in being one of the most acknowledged astronomers in the mid-1990's ${ }^{2}$. Bohdan was always honest, and ready to accept any (rare) mistakes with highest integrity. I still remember a postdoc talking to me about some mistakes he found in Bohdan's interpretation of the stellar populations in the Galactic bulge. The postdoc was greatly relieved when Bohdan accepted his mistake graciously and strongly encouraged him to publish and even advised him on how to best present his results. In my eyes,

\footnotetext{
${ }^{2}$ ftp://gradj.pa.uky.edu//dima//aai//top95.txt
} 
this made him a greater man and scientist.

Bohdan was not only a great scientist, but also a wonderful man. He was, as in science, very generous. Perhaps because we were both emigrants from communist countries (Poland and China), we shared some of the same bureaucratic problems (e.g., on visas). He gave me sound advice on the issues, both when I was his student and afterwards. In life, Bohdan was simple and unpretentious. My impression is that he was a man totally devoted to science, but also derived much pleasure in simple discoveries in daily life. In the few years after he was diagnosed with cancer, he was still optimistic, doing science in "his borrowed time."

As I am writing this tribute, I have many memories and feelings that are difficult to put down in words. From his many emails to me, I can almost hear him talking. His handwritings on my note pads reminded me of many occasions when we sat face-to-face in his office, discussing projects during which he would say "let me draw a simple picture." With his passing, I have lost my personal mentor and collaborator, and the world has lost "a great scientist and a wonderful man" [2].

\section{References}

[1] Alcock, C., Paczynski, B. 1979, Nature, 281, 358

[2] Draine, B. T., Ostriker, J. P. 2007, Nature, 447, 1064

[3] Mao, S., Paczyński, B. 1991, ApJ, 374, L37

[4] Mao, S., Paczyński, B. 1992, 388, L45

[5] Meegan, C. A. et al. 1992, Nature, 353, 143

[6] Paczyński, B. 1970, AcA, 20, 47

[7] Paczyński, B. 1971, AR\&AA, 9, 183

[8] Paczyński, B., Wiita, P. J. 1980, A\&A, 88, 23

[9] Paczyński, B. 1986, ApJ, 304, 1

[10] Paczyński, B. 1986, ApJ, 308, 43

[11] Paczyński, B. 1987, Nature, 325, 572

[12] Paczyński, B. 1998, ApJ, 494, L45 